\title{
THE CENTRAL REGION OF THE BARRED SPIRAL GALAXY NGC 1097 PROBED BY AKARI NEAR-INFRARED SPECTROSCOPY
}

\author{
T. Kondo ${ }^{1}$, H. Kaneda ${ }^{1}$, S. Oyabu ${ }^{1}$, D. Ishinara ${ }^{1}$, T. Mori ${ }^{1}$, M. Yamagishi $^{1}$, T. Onaka $^{2}$, I. Sakon $^{2}$, \\ AND T. SUZUKI ${ }^{3}$ \\ ${ }^{1}$ Graduate School of Science, Nagoya University, Chikusa-ku, Nagoya 464-8602, Japan \\ ${ }^{2}$ Graduate School of Science, The University of Tokyo, Bunkyo-ku, Tokyo 113-0033, Japan \\ ${ }^{3}$ Institute of Space and Astronautical Science, Japan Aerospace Exploration Agency, Sagamihara, Kanagawa \\ 252-5210, Japan \\ E-mail:kondo@u.phys.nagoya-u.ac.jp \\ (Received June 30, 2012; Accepted August 15, 2012)
}

\begin{abstract}
With AKARI, we carried out near-infrared spectroscopy of the nearby barred spiral galaxy, NGC 1097, categorized as Seyfert 1 with a circumnuclear starburst ring. Our observations mapped the galactic center region. As a result, we obtain the spatial distributions of the polycyclic aromatic hydrocarbon $3.3 \mu \mathrm{m}$ and the aliphatic hydrocarbon 3.4-3.6 $\mu \mathrm{m}$ emission. The former is detected from all the observed regions and the latter is enhanced near the bar connecting the ring with the nucleus. In addition, we detect absorption features due to $\mathrm{H}_{2} \mathrm{O}$ ice and $\mathrm{CO} / \mathrm{SiO}$ at the ring and the galactic center, while we detect the hydrogen recombination line Br $\alpha$ only from the ring. Hence the observed spectra change dramatically within the central $1 \mathrm{kpc}$ region.
\end{abstract}

Key words: galaxies; individual (NGC 1097): galaxies; ISM: galaxies; nuclei: galaxies; starburst: infrared; galaxies

\section{INTRODUCTION}

NGC 1097 is a nearby (19.1 Mpc; Willick et al., 1997) barred spiral galaxy with a starburst ring of $\sim 2 \mathrm{kpc}$ in diameter and categorized as Seyfert 1. The optical and near-infrared (near-IR) images reveal the dust lanes running along the primary bar $(\sim 15 \mathrm{kpc}$ in length) outside the ring and the second bar $(\sim 1 \mathrm{kpc})$ inside the ring (Quillen et al., 1995). It is suggested that gas in the ring is flowing into the nucleus along the second bar (Prieto et al., 2005).

We carried out the near-IR $(2.5-5.0 \mu \mathrm{m})$ slit spectroscopic observations of a central region of NGC 1097 with the IRC on board the AKARI (Kondo et al., 2012). Our observations mapped the area of a half of the ring and the galactic center $\left(\sim 50^{\prime \prime} \times 10^{\prime \prime}\right)$ with the resolution of $\sim 5^{\prime \prime}$. Figure 1 shows the slit positions of two observations and the slit sub-aperture regions, from which we created the spectra in Figure 2.

\section{RESULT AND DISCUSSION}

The spectra in Figure 2 show polycyclic aromatic hydrocarbon (PAH) emission at $3.3 \mu \mathrm{m}$, aliphatic subfeatures at $3.4-3.6 \mu \mathrm{m}, \operatorname{Br} \alpha$ emission at $4.05 \mu \mathrm{m}$, and broad absorption features due to $\mathrm{H}_{2} \mathrm{O}$ ice at $3.05 \mu \mathrm{m}$ and blending of $\mathrm{SiO}$ and $\mathrm{CO}$ at $4.30 \mu \mathrm{m}$ and $4.66 \mu \mathrm{m}$, respectively. We fit the continuum by a power-law function, while the emission lines/features and the absorption features are fitted by Gaussian or Lorentzian profiles.

We calculate the fluxes of the spectral components and create the two-dimensional spectral maps shown in Figure 3. The PAH $3.3 \mu \mathrm{m}$ emission feature shows a relatively uniform distribution in the starburst ring. Since the Br $\alpha$ emission shows a local peak in the eastern part of the ring, the starburst activity is likely to be enhanced locally in this region (Kondo et al., 2012). The 3.4-3.6 $\mu \mathrm{m}$ sub-features are strong along the sec- 


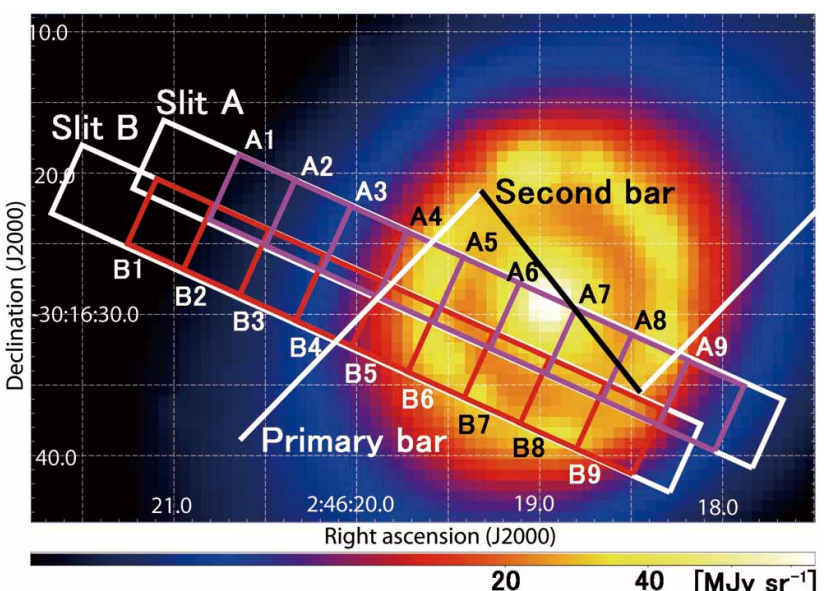

Fig. 1. Positions of the two slit apertures and their sub-apertures (small boxes) used to create the spectra, overlaid on the Spitzer/IRAC $3.6 \mu \mathrm{m}$ image.
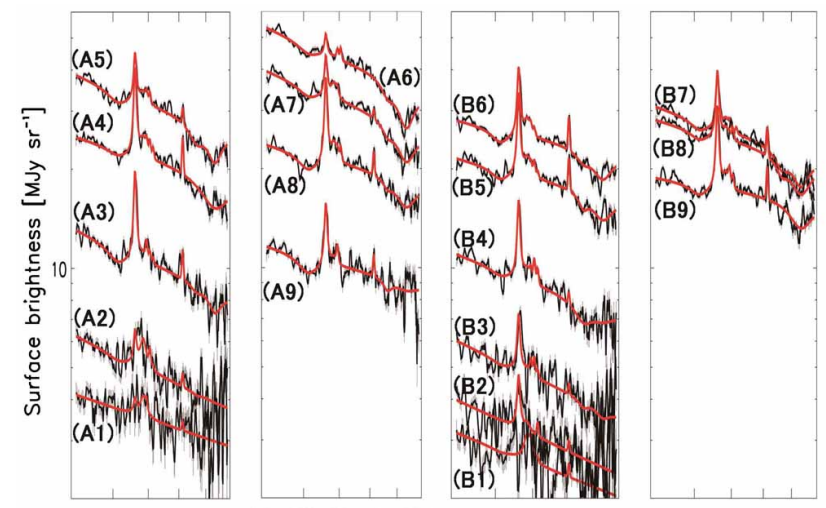

$\begin{array}{llllllllllllllllllll}2.5 & 3.0 & 3.5 & 4.0 & 4.55 .0 & 2.5 & 3.0 & 3.5 & 4.04 .55 .0 & 2.5 & 3.0 & 3.5 & 4.0 & 4.55 .0 & 2.5 & 3.0 & 3.5 & 4.0 & 4.55 .0 \\ \text { Wavelength }[\mu \mathrm{m}]\end{array}$

Fig. 2. AKARI/IRC 2.5-5.0 $\mu \mathrm{m}$ spectra together with the names of the slit sub-apertures indicated in Figure 1. For each spectrum, the solid curve indicates the best-fit model.

ond bar, which suggests that shattering of carbonaceous grains has occured because of turbulent motion of gas (Jones et al., 1996). The equivalent width (EW) of the $\mathrm{CO} / \mathrm{SiO}$ absorption is almost constant over the central region. Since the $\mathrm{CO} / \mathrm{SiO}$ absorption is probably of low-mass O-rich star origin (Cohen et al., 1992), old stellar populations are likely to be dominant over the central region. There is no contribution of hot dust at the center, because the EW does not decrease toward the center. There seems to be no starburst activity at the center, since the $\operatorname{Br} \alpha$ emission is not significantly detected. Hence we cannot find any evidence for the presence of nuclear activity from our observations.
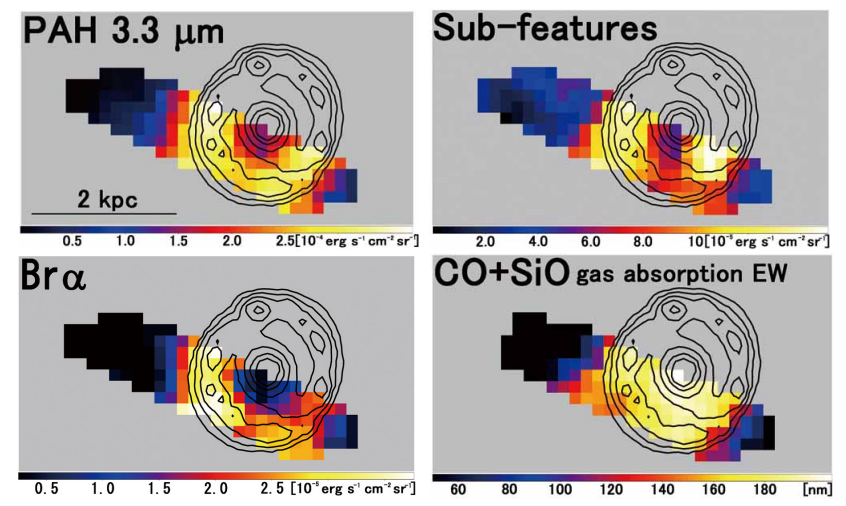

Fig. 3. Spectral maps shown together with the contour map of the Spitzer/IRAC $3.6 \mu \mathrm{m}$ band (Figure 1). The contours are drawn at logarithmically-spaced six levels from $50 \%$ to $15 \%$ of the peak.

\section{REFERENCES}

Cohen, M., et al., 1992, Spectral Irradiance Calibration in the Infrared. III - The Influence of CO and SiO, AJ, 104, 2045

Jones, A. P., Tielens, A. G. G. M., \& Hollenbach, D. J., 1996, Grain Shattering in Shocks: The Interstellar Grain Size Distribution, ApJ, 469, 740

Kondo, T., et al., 2012, The Central Region of the Barred Spiral Galaxy NGC 1097 Probed by AKARI Near-infrared Spectroscopy, ApJ, 751, L18

Prieto, M. A., Maciejewski, W., \& Reunanen, J., 2005, Feeding the Monster: The Nucleus of NGC 1097 at Subarcsecond Scales in the Infrared with the Very Large Telescope, AJ, 130, 1472

Quillen, A. C., Frogel, J. A., Kuchinski, L. E., \& Terndrup, D. M., 1995, Multiband Images of the Barred Galaxy NGC 1097, AJ, 110, 156

Willick, J. A., et al., 1997, Homogeneous VelocityDistance Data for Peculiar Velocity Analysis. III. The Mark III Catalog of Galaxy Peculiar Velocities, ApJS, 109, 333 